\title{
Metodología experimental para determinar los parámetros del proceso de moldeo por compresión de materiales compuestos de termoplástico reciclado y madera recuperada
}

\section{An experimental methodology to determine the parameters of the compression molding process of composite materials made of recycled thermoplastic and recovered wood}

Walter Tupia ${ }^{1}$, Luz Elena Brañez Haro ${ }^{1}$, Adan Arribasplata ${ }^{1}$, Julio Acosta ${ }^{1}$

\begin{abstract}
${ }^{1}$ Sección Ingeniería Mecánica, Departamento de Ingeniería, Pontificia Universidad Católica del Perú, San Miguel, Lima, Peru.

e-mail: wtupia@pucp.edu.pe, luz.branez@pucp.edu.pe, adan.arribasplata@pucp.pe,jacosta@pucp.edu.pe
\end{abstract}

\begin{abstract}
RESUMEN
El objetivo de esta investigación es determinar los parámetros del proceso de sinterizado de materiales compuestos plástico-madera (CPM) fabricados mediante moldeo por compresión y con mejores propiedades desde el punto de vista de ingeniería. La estructura de este artículo es, en sí misma, una propuesta de metodología experimental para obtener CPM a partir de partículas de termoplásticos reciclados y maderas recuperadas. Ésta incluye la caracterización de los materiales y pruebas de moldeo por compresión, para determinar en una primera etapa la temperatura y tiempo de sinterizado y, en una segunda, el contenido y tamaño de las partículas de madera. Empleando madera capirona recuperada (MCR) y polipropileno reciclado (PPR), se evalúan dichos parámetros mediante ensayos en muestras moldeadas. Para este caso, los parámetros hallados son: 50 min de permanencia en horno a $190{ }^{\circ} \mathrm{C}, 30 \%$ y $70 \%$ en volumen de MCR y PPR respectivamente y partículas de MCR de $0,98 \mathrm{~mm}$ a $1,90 \mathrm{~mm}$ de tamaño.
\end{abstract}

Palabras clave: compuestos plástico-madera, moldeo por compresión, reciclado, recuperado, sinterizado.

\begin{abstract}
The objective of this research is to determine the sintering process parameters of wood-plastic composite materials (WPC) manufactured by compression molding and with better properties from an engineering point of view. The structure of this article is, by itself, a proposal of experimental methodology to obtain WPC from recycled thermoplastic particles and recovered wood. This methodology includes the characterization of both starting materials and compression-molding tests, to determine in a first stage the temperature and time of sintering and, in a second stage, the content and size of the wood particles. Using recovered capirona wood (RCW) and recycled polypropylene (RPP); these parameters are evaluated by standardized tests on molded samples. For this case, the parameters are: $50 \mathrm{~min}$ of time into the oven at $190{ }^{\circ} \mathrm{C}, 30 \%$ and $70 \%$ in volume of RCW and RPP respectively and RCW particles from $0,98 \mathrm{~mm}$ to $1,90 \mathrm{~mm}$ of size.
\end{abstract}

Keywords: wood-plastic composites, compression molding, recycled, recovered, sintering.

\section{INTRODUCCIÓN}

Los procesos para la obtención de materiales compuestos plástico-madera son diversos, siendo la extrusión y el moldeo por inyección los más utilizados porque permiten obtener productos de forma compleja con altos volúmenes de producción, 
aunque con la restricción de usar madera en forma de polvo. Como consecuencia, en la actualidad existe una gran cantidad de investigaciones, literatura y productos comerciales obtenidos con estos procesos $[1,2]$. No obstante, para fabricar componentes de geometría más simple, el proceso de moldeo por compresión es una alternativa económica al permitir el empleo de partículas de madera de mayor tamaño. Además, la presión de trabajo es mucho más uniforme en el interior del molde favoreciendo el proceso de conformado y sinterizado, lo cual es una ventaja frente a los otros procesos mencionados, ya que una presión uniforme es deseable para evitar daños en el refuerzo [3]. Sin embargo, hasta el momento no existen muchos trabajos de investigación orientados al estudio del moldeo por compresión de CPM, del sinterizado del plástico en presencia de la madera ni al de sus parámetros.

El proceso de sinterizado es importante porque influirá en las propiedades del material compuesto. Se trata de un proceso térmico, gracias al cual las partículas pasan de ser un sistema de elementos individuales a formar finalmente un sólido con propiedades que serán resultado de la manera en la que se ha realizado dicho proceso [4]. Presenta tres etapas; la primera, llamada de "formación de cuellos", es la etapa en que se inicia el crecimiento de las zonas de contacto entre las partículas con la consiguiente reducción de la superficie libre de las mismas. La etapa intermedia es la densificación; en ésta, luego de un crecimiento intensivo de los cuellos, las partículas comienzan a perder su individualidad y se forma una red compleja de poros que se van cerrando. La densificación es la etapa más importante de todo el proceso porque en ésta quedan determinadas las propiedades del material. La etapa final se inicia cuando la mayor parte de los poros se cierran y van quedando aislados entre sí tomando forma esferoidal. Existe la posibilidad de que queden poros remanentes después del proceso debido a que los gases generados durante el calentamiento no pudieron eliminarse quedando encapsulados [5]. En la Figura 1 se puede apreciar una microfotografía de dos partículas aisladas de polipropileno durante las etapas del sinterizado, desde la formación del cuello hasta la coalescencia.
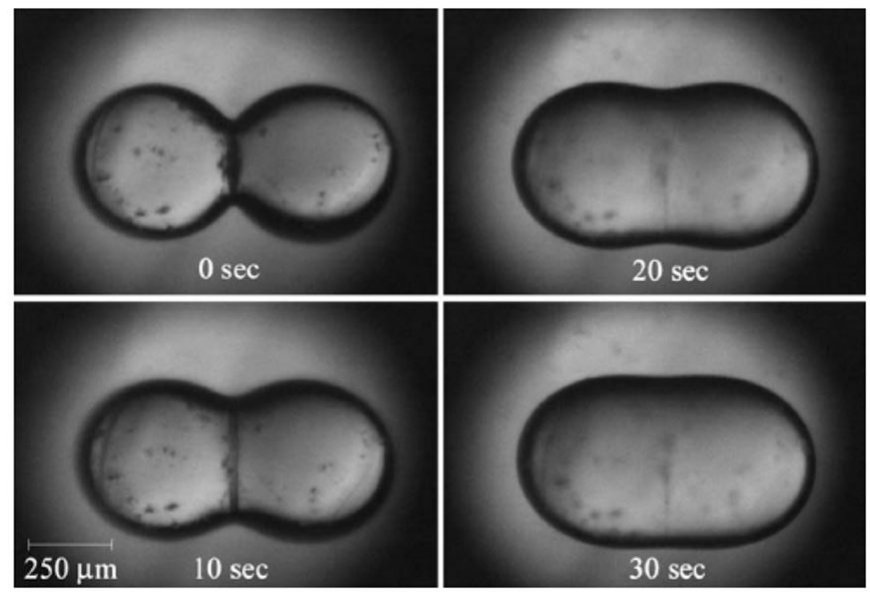

Figura 1: Microfotografía óptica de coalescencia de partículas de polipropileno [6] which equated the work of surface tension to the work done by viscous stresses while assuming biaxial extensional flow kinematics, was extended to the case of time-dependent material functions using the Upper Convected Maxwell (UCM.

En esta perspectiva, el objetivo principal del presente trabajo es definir una metodología para determinar los parámetros que permitan obtener las mejores propiedades mecánicas en los materiales compuestos plástico-madera (CPM) obtenidos a partir de la mezcla de partículas de estos constituyentes y mediante moldeo por compresión. Con ello se busca volver a darle valor industrial a los materiales reciclables, como son los termoplásticos, y a aquellos que pueden ser recuperados de procesos industriales como es el caso de la madera. En este trabajo se utilizará, de manera particular y como ejemplo de aplicación, polipropileno y madera capirona (calycophyllum spruceanum) especie oriunda del Perú.

\section{MATERIALES Y MÉTODOS}

La metodología propuesta en este estudio presenta cuatro etapas. En la primera etapa se caracterizan los materiales a emplear en la elaboración del CPM; en la segunda se moldean muestras del material compuesto y se fabrican probetas a partir de dichas muestras; en la tercera se realizan ensayos normalizados para la determinación de las propiedades del CPM y finalmente la cuarta etapa corresponde al análisis morfológico del material compuesto. Los ensayos se realizan según normas técnicas internacionales, principalmente las ASTM. 


\subsection{Caracterización de los materiales}

\subsubsection{Madera recuperada}

Inicialmente las partículas de madera se deben clasificar por su tamaño. Para ello se emplean tamices con mallas de acero inoxidable. A dichos tamices se les asigna una denominación (MR1, MR2, MR3, MR4 y MR5) correspondiente a la malla normalizada de acuerdo con la norma ASTM E11-15, la que a su vez está relacionada con el máximo tamaño de partícula que pueden retener, tal como se aprecia en la Tabla 1.

Tabla 1: Serie de mallas utilizadas en la clasificación por tamaño de las partículas de madera.

\begin{tabular}{c|c|c}
\hline DENOMINACIÓN & MALLA NORMALIZADA (ASTM) & TAMAÑO DE PARTíCULA (TP) \\
\hline MR1 & MESH 3 1/2 & $3,33 \mathrm{~mm}<\mathrm{TP} \leq 5,15 \mathrm{~mm}$ \\
\hline MR2 & MESH 6 & $1,90 \mathrm{~mm}<\mathrm{TP} \leq 3,33 \mathrm{~mm}$ \\
\hline MR3 & MESH 10 & $0,98 \mathrm{~mm}<\mathrm{TP} \leq 1,90 \mathrm{~mm}$ \\
\hline MR4 & MESH 18 & $0,48 \mathrm{~mm}<\mathrm{TP} \leq 0,98 \mathrm{~mm}$ \\
\hline MR5 & MESH 35 & $\mathrm{TP} \leq 0,48 \mathrm{~mm}$ \\
\hline
\end{tabular}

Mediante el tamizado se clasifican las partículas en los grupos correspondientes a cada malla; así, al referirse a partículas MR2, significa que en esta malla se retienen partículas con tamaños mayores a 1,90 $\mathrm{mm}$ hasta 3,33 mm inclusive.

Otro aspecto importante por determinar son las propiedades fisicoquímicas de la madera, como su densidad, contenido de humedad, de absorción de agua, temperatura de degradación y además sus propiedades mecánicas. Los ensayos a realizar se encuentran listados en la Tabla 2.

Tabla 2: Ensayos para la caracterización de la madera.

\begin{tabular}{c|c}
\hline ENSAYO & NORMA ASTM \\
\hline Densidad & D2395-17 \\
\hline Contenido de humedad & E871-82 (2013) \\
\hline Absorción de agua & D1037-12 \\
\hline Temperatura de degradación & E1131-08 (2014) \\
\hline Flexión & D1037-12 \\
\hline
\end{tabular}

Es preciso identificar las propiedades mecánicas relacionadas con las posibles aplicaciones de los materiales compuestos plástico-madera para seleccionar los ensayos mediante los cuales se determinen las propiedades de la madera y compararlas más adelante con las del CPM [7]. En consecuencia, en este trabajo, se ha seleccionado el ensayo de flexión para determinar la resistencia mecánica y el módulo elástico en flexión.

\subsubsection{Termoplástico reciclado}

El termoplástico reciclado debe ser previamente clasificado y luego picado para poder utilizarlo como material constitutivo del CPM.

Las características más importantes del termoplástico que se deben conocer son: identificación del tipo de termoplástico, índice de fluidez, temperaturas de fusión, transición vítrea y de degradación, así como sus propiedades mecánicas.

Con el fin de determinar o verificar el tipo de termoplástico reciclado, se realiza un análisis de espectroscopía infrarroja por transformada de Fourier. Se utiliza un espectroscopio, marca Bruker Tensor 27, y con ayuda del software del equipo se observa el espectro de los grupos funcionales del polímero en estudio para identificarlo por comparación con patrones. 
Tabla 3: Ensayos para caracterización del termoplástico.

\begin{tabular}{c|c}
\hline ENSAYO & NORMA ASTM \\
\hline Índice de fluidez & D1238-13 \\
\hline Temperatura de fusión/transición vítrea & D3418-12 \\
\hline Temperatura de degradación & ASTM E1131-08 (2014) \\
\hline
\end{tabular}

Los ensayos aplicados para la determinación de las propiedades del polímero termoplástico están indicados en la Tabla 3.

Para determinar el índice de fluidez del termoplástico se utilizó el equipo Zwich/Roell Cflow, que permite calentar el polímero y extruirlo durante un periodo de tiempo determinado. Para el ensayo de calorimetría diferencial de barrido (DSC) se utilizó el equipo Júpiter STA 449 F3 - Netzsch que trabaja con atmósfera inerte controlada en un rango de temperaturas entre $150^{\circ} \mathrm{C}$ y $2400^{\circ} \mathrm{C}$.

Igual que en el caso de la madera, es importante conocer las propiedades mecánicas del termoplástico reciclado a emplear en la elaboración del CPM. Para este trabajo de investigación se han tomado como referencia las propiedades del polipropileno empleado en la fabricación de las tapas con las que se ha hecho la simulación del reciclado. Sin embargo, al tratarse de materiales reciclados, se debe considerar que éstos han sufrido transformaciones físicas durante los procesos de fabricación y, en consecuencia, sus propiedades mecánicas disminuyen. No obstante, se recomienda moldear por compresión el termoplástico reciclado para obtener probetas y realizar los ensayos correspondientes.

\subsection{Diseño experimental}

Es preciso definir los parámetros principales del proceso de moldeo por compresión aplicado a la fabricación de CPM, tales como: temperatura y tiempo de calentamiento, tamaño de partículas de madera y proporción de termoplástico-madera.

La experimentación tiene dos partes; el objetivo de la Parte I es determinar la temperatura de calentamiento y el tiempo de permanencia en el horno para llevar a cabo el proceso de sinterizado del CPM, de tal manera que se obtengan las mejores propiedades en el material compuesto y, con el mismo propósito, la Parte II tiene por finalidad definir los otros parámetros: tamaño de partículas de madera a emplear y proporción termoplástico-madera.

Durante el desarrollo de la Parte I se mantiene fija la proporción termoplástico-madera y el tamaño de partícula. La mezcla se mantiene en todas las pruebas en una proporción 70\%-30\% en volumen de plástico-madera respectivamente, la cual se ha definido a partir de pruebas preliminares de moldeo, comprobándose que una cantidad de polímero mayor que la de madera facilita el moldeo. Además, se emplean partículas de tamaño MR2, mayor que el polvo de madera, porque se busca conocer las posibilidades del moldeo por compresión como una alternativa a la inyección y a la extrusión, que utilizan polvo de madera y requieren una mayor inversión en equipos.

Así mismo, las partículas de madera se utilizan sin tratamiento previo y evitando el uso de aditivos, en coherencia con el cuidado del ambiente.

La temperatura de calentamiento debe evaluarse en un rango que incluya la temperatura de fusión del termoplástico; adicionalmente, la temperatura empleada no debe exceder el rango de temperaturas de degradación del tipo de madera utilizada (por lo general entre los $200^{\circ} \mathrm{C}$ y $220^{\circ} \mathrm{C}$ ), puesto que ello podría perjudicar las propiedades del material compuesto. Se recomienda comenzar las pruebas con la mayor temperatura de calentamiento, por debajo de la de degradación, a fin de evaluar el sinterizado a diferentes tiempos de permanencia en el horno; luego, basados en las observaciones de la integridad estructural de las muestras obtenidas a esta temperatura, de manera análoga se podrán definir los rangos de tiempo correspondientes a las temperaturas menores del estudio.

En resumen, en esta etapa se evaluarán: una proporción plástico-madera, un tamaño de partícula, 3 a 4 temperaturas y 5 tiempos de permanencia en horno para cada temperatura. Se fabricarán 3 muestras planas rectangulares para cada combinación de estos parámetros, obteniéndose un total de 45 a 60 muestras como se puede ver en la Tabla 4.

Tabla 4: Total de muestras para el moldeo (Parte I)

\begin{tabular}{c|c|c|c|c|c}
\hline $\begin{array}{c}\% \\
\% \mathbf{P}\end{array}$ & TAMAÑO & TEMP. & TIEMPO & $\begin{array}{c}\text { MUESTRAS A LAS } \\
\text { MISMAS CONDICIONES }\end{array}$ & $\begin{array}{c}\text { TOTAL DE } \\
\text { MUESTRAS }\end{array}$ \\
\hline 1 & 1 & $3 \mathrm{a} 4$ & 5 & 3 & $45 \mathrm{a} 60$ \\
\hline
\end{tabular}


En la Parte II se utiliza la temperatura y el tiempo de calentamiento definidos en la Parte I, se varía el tamaño de partículas de madera y la proporción en volumen de los constituyentes. En este sentido, se evalúan 3 diferentes porcentajes de constituyentes del material compuesto además del termoplástico puro (0, 30, 40 y $50 \% \mathrm{MR})$ y 3 tamaños de partículas (MR1, MR2 y MR3). Considerando 5 muestras para cada condición se obtiene un total de 50 muestras, como se puede apreciar en la Tabla 5.

Tabla 5: Total de muestras para el moldeo (Parte II)

\begin{tabular}{c|c|c|c|c|c}
\hline \multicolumn{2}{c|}{ CONSTITUYENTES } & \multicolumn{5}{c}{ NÚMERO DE MUESTRAS A OBTENER } \\
\hline$\% P R$ & $\% M R$ & MR1 & MR2 & MR3 & TOTAL \\
\hline 100 & 0 & 5 & 5 & 5 & 5 \\
\hline 70 & 30 & 5 & 5 & 5 & 15 \\
\hline 60 & 40 & 5 & 5 & 5 & 15 \\
\hline 50 & 50 & 5 & TÓstico puro) & 5 \\
\hline \multicolumn{7}{c|}{ TOTAL DE MUESTRAS } \\
\hline
\end{tabular}

\subsection{Moldeo de muestras y fabricación de probetas}

Las muestras planas rectangulares de $300 \mathrm{~mm}$ x $150 \mathrm{~mm}$ x $10 \mathrm{~mm}$ se fabrican utilizando moldes de aluminio y un sistema de moldeo por compresión diseñado y fabricado específicamente para este trabajo. El sistema está constituido por un horno eléctrico de $12 \mathrm{~kW}$ y temperatura máxima de servicio de $450^{\circ} \mathrm{C}$, una prensa hidráulica de 100 toneladas y un mecanismo de transporte del molde que permite el traslado al horno y retorno a la prensa para el enfriamiento bajo presión, así como la extracción de la muestra.

\subsubsection{Cantidad de partículas de madera y de termoplástico por muestra.}

Para cada muestra, la cantidad de partículas de madera y termoplástico se determina con las ecuaciones (1), (2) y (3).

$$
\begin{gathered}
m_{M}=V_{T m} \times\left(\frac{\% V_{M}}{100}\right) \times \rho_{M} \\
m_{P}=V_{T m} \times\left(\frac{\% V_{P}}{100}\right) \times \rho_{P} \\
m_{T}=m_{M}+m_{P}
\end{gathered}
$$

En estas expresiones, $\mathrm{m}_{\mathrm{M}} \mathrm{y} \mathrm{m}_{\mathrm{P}}$ son las masas de la madera y del plástico, $\% \mathrm{~V}_{\mathrm{M}} \mathrm{y} \% \mathrm{~V}_{\mathrm{P}}$ son los porcentajes en volumen de la madera y del plástico, $\rho_{\mathrm{M}}$ y $\rho_{\mathrm{P}}$ son las densidades de la madera y el plástico respectivamente, $\mathrm{m}_{\mathrm{T}}$ y $\mathrm{V}_{\mathrm{Tm}}$ son la masa y el volumen total de la muestra. Con ellas se calculan las masas de las partículas de cada material a emplear en la fabricación de las muestras del CPM.

Se recomienda considerar un 10\% adicional de la masa total por pérdidas durante el proceso de compresión, principalmente debido a las rebabas. Con las masas determinadas, se procede al pesaje de la madera y el plástico correspondiente a cada muestra.

\subsubsection{Mezclado, homogenización y preparación del molde}

Las partículas de ambos materiales se mezclan en un recipiente agitando durante 2 minutos hasta que se consiga una mezcla homogénea.

Con la finalidad de facilitar la extracción de la muestra del material compuesto, se debe limpiar el molde y aplicar un desmoldante de silicona en las paredes del interior de la cavidad y en la parte inferior de la tapa.

Luego, el molde se precalienta en el horno hasta que alcance una temperatura cercana a la deseada para el moldeo, lo cual permitirá disminuir el tiempo de calentamiento de la mezcla. El control de dicha temperatura se realiza con una termocupla en contacto con el molde. 


\subsubsection{Carga de la mezcla y cierre del molde}

Una vez precalentado el molde, se retira del horno y se deposita la mezcla en éste como se ve en la Figura 2(a); a continuación, se coloca la tapa mostrada en la Figura 2(b) y se conecta la termocupla de contacto para el registro de la temperatura del molde.

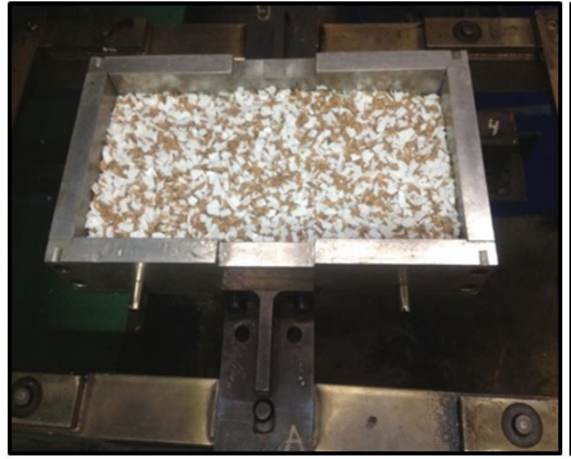

(a)

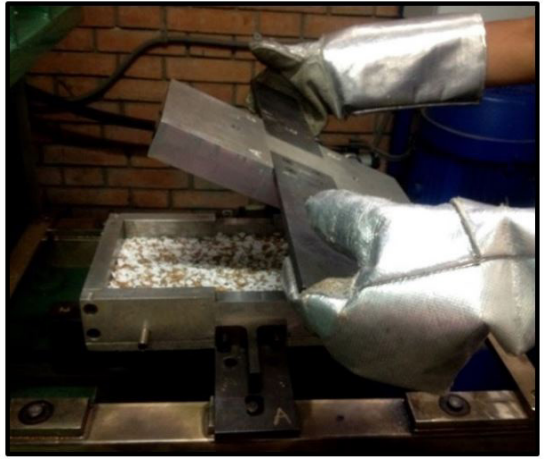

(b)

Figura 2: Carga y cierre del molde: a) mezcla homogénea de partículas en el molde y b) colocación de la tapa.

\subsubsection{Calentamiento en el horno}

Con ayuda del mecanismo de transporte, que consiste en un carro que se desplaza sobre rieles, se lleva el molde conteniendo la mezcla al interior del horno para el calentamiento. La temperatura de calentamiento del molde y la del interior del horno es registrada con la ayuda de termocuplas; se tiene especial cuidado con el control de los distintos tiempos de permanencia en el horno para cada temperatura de prueba.

\subsubsection{Enfriamiento bajo presión}

Cumplido el tiempo de permanencia en el horno, se procede a retirar el molde y se traslada hacia la prensa hidráulica. Luego se le aplica una fuerza de $34 \mathrm{kN}$ mediante el vástago de la prensa durante 10 minutos, a la vez que se lleva a cabo el enfriamiento con aire forzado.

\subsubsection{Extracción y rotulado de las muestras}

Terminada la etapa de compresión y enfriamiento, se procede a extraer la muestra del molde por medio de un juego de pines extractores. Finalmente, se retiran las rebabas y se etiquetan las muestras de acuerdo con las diferentes condiciones y parámetros empleados en el proceso de fabricación.

\subsubsection{Fabricación de probetas para los ensayos}

Para determinar las propiedades del material compuesto se fabrican las probetas necesarias para los ensayos de flexión, tracción, densidad y absorción de agua según las normas ASTM correspondientes.

En la Parte I de la experimentación se ha elegido el ensayo de flexión, como ensayo de control, con el fin de establecer la temperatura de calentamiento y el tiempo de permanencia en el horno de tal manera que dichos parámetros correspondan a las muestras de mejores propiedades mecánicas. La elección de este ensayo se debe principalmente a que la flexión es el tipo de solicitación más probable de los productos fabricados con CPM en función a las aplicaciones [7]. Además, se trata de un ensayo sencillo en el que las probetas son simples, fáciles de fabricar y de bajo costo.

De cada muestra se obtienen dos probetas de flexión, por tanto $6(3 \times 2)$ probetas de CPM fabricadas a las mismas condiciones de temperatura de calentamiento y tiempo de permanencia en el horno; obteniendo un total de 90 (45 x 2) a 120 (60 x 2) probetas de flexión a ensayar, según se puede deducir de la Tabla 4.

En la Parte II, se requiere obtener una probeta de flexión, una de tracción y cuatro probetas para los ensayos de densidad y de absorción de agua por cada muestra. A partir de esta información se deduce que, para cada condición de temperatura / tiempo / proporción de plástico-madera / tamaño de partícula, se ensayan las siguientes cantidades de probetas: 5 de flexión, 5 de tracción, 20 de densidad y 20 de absorción de agua. El total de probetas es: 50 (50 x 1) probetas de flexión, $50(50 \times 1)$ de tracción, $200(50$ x 4) de densidad y 200 (50 x 4) de absorción de agua, como se puede deducir de la Tabla 5. 


\subsection{Ensayos}

Para la caracterización del CPM se realizaron estudios térmicos y morfológicos, así como ensayos para determinar sus propiedades físicas (Tabla 6).

Con el propósito de analizar la adhesión de las partículas de madera y plástico, se realizan los estudios térmicos y morfológicos en las zonas de fractura de las probetas luego de los ensayos de tracción y flexión, utilizando un microscopio de barrido electrónico (SEM) Quanta 650, operado con un voltaje de aceleración de $15 \mathrm{kV}$ y en modo de bajo vacío, además de los equipos ya mencionados en el acápite 2.1.2 para la realización del TGA y el DSC.

Tabla 6: Ensayos para la caracterización del CPM.

\begin{tabular}{l|c}
\hline \multicolumn{1}{c|}{ ENSAYO } & NORMA ASTM \\
\hline Densidad & D2395-17 \\
\cline { 1 - 1 } Absorción de agua & D1037-12* \\
\cline { 1 - 2 } Flexión & \\
\hline Tracción & \\
\hline
\end{tabular}

* Norma específica para madera y materiales compuestos con madera como constituyente.

\section{RESULTADOS Y DISCUSIÓN}

A continuación, se presentan los resultados para el caso del material compuesto fabricado a partir de polipropileno reciclado (PPR) y madera capirona recuperada (MCR) mediante moldeo por compresión para la validación de la metodología propuesta en este trabajo.

La madera capirona proviene de las mermas de una fábrica de muebles. Dichos residuos recuperados, en forma de partículas, provienen del proceso de torneado de partes de muebles.

Se ha visto conveniente simular el reciclado de tapas blancas de botellas para bebidas gasificadas sin sello, las cuales fueron picadas para su utilización en el moldeo. Ello con el fin de evitar la inclusión de otras variables de las etapas previas del reciclado del polipropileno, tales como segregación, lavado y secado.

\subsection{Caracterización de la madera capirona}

Luego de los ensayos para la determinación de las propiedades físicas de la capirona se obtuvieron los resultados mostrados en la Tabla 7.

Tabla 7: Propiedades físicas de la madera capirona.

\begin{tabular}{l|c}
\hline \multicolumn{1}{c|}{ PROPIEDAD } & VALOR HALLADO \\
\hline Densidad & $0,74 \pm 0,02 \mathrm{~g} / \mathrm{cm}^{3}$ \\
\hline Contenido de humedad & $12,8 \pm 0,2 \%$ \\
\hline Absorción de agua & $42,8 \pm 8,4 \%$ \\
\hline Temp. de inicio de degradación & $240^{\circ} \mathrm{C}$ \\
\hline
\end{tabular}

Trabajos anteriores de otros investigadores presentan resultados muy similares a los obtenidos en densidad [8,9], contenido de humedad [10] y absorción de agua [11].

Según el análisis termogravimétrico de la capirona, se pudo observar la degradación de la madera desarrollada en dos etapas. La primera etapa ocurre hasta $\operatorname{los} 125^{\circ} \mathrm{C}$ aproximadamente, con una pérdida de humedad del $13 \%$ en masa de la madera, de acuerdo con el resultado del ensayo de absorción de humedad. La segunda etapa se produce en un rango de temperatura de $240^{\circ} \mathrm{C}$ a $600^{\circ} \mathrm{C}$ aproximadamente con una pérdida de masa de $64 \%$ que probablemente involucre en mayor proporción la degradación de la lignina, hemicelulosa y celulosa [12]. La proporción remanente se degrada a temperaturas mayores a $600^{\circ} \mathrm{C}$. A partir de este resultado se deduce que, para la fabricación del CPM, no se deben emplear temperaturas mayores a $240^{\circ} \mathrm{C}$ con el fin de evitar el inicio de la degradación. 
Los valores de las propiedades en flexión de la capirona de la Tabla 8 se presentan como referencia para compararlos más adelante con los resultados de los ensayos del CPM.

Tabla 8: Resultados del ensayo de flexión aplicado a probetas de madera capirona [13].

\begin{tabular}{c|c|c}
\hline DIRECCIÓN DE LA FIBRA & $\mathbf{R}_{\mathbf{f ~ P R O M}}$ (MPa) & $\mathbf{E}_{\mathbf{f P R O M}}(\mathbf{G P a})$ \\
\hline Longitudinal & $128 \pm 18,2$ & $13,5 \pm 2,6$ \\
\hline Transversal & $8 \pm 2,6$ & $1,3 \pm 0,2$ \\
\hline
\end{tabular}

\subsection{Caracterización del polipropileno}

Por medio de la espectroscopía FTIR se pudieron observar los picos característicos en los números de onda, tanto para el PP-virgen y el PPR correspondientes a las bandas características de las vibraciones de los enlaces $\mathrm{C}$-H en el polipropileno y aquellos correspondientes a las deformaciones asimétrica y simétrica del $\mathrm{CH}_{3}$ [14]. De todo ello se puede confirmar que el polímero reciclado es un polipropileno, aunque se observó cierta reducción de la transmitancia comparada con el PP-virgen debido a la degradación sufrida a través del proceso de moldeo por inyección cuando se le transformó en tapas.

En la Tabla 9 se muestran los principales resultados de las pruebas y ensayos realizados al PPR. Como era de esperarse, el índice de fluidez del PPR obtenido es mayor que el del PP-virgen (2,6 g/10 min), siendo aún adecuado para moldeo por compresión [15]. El material reciclado se degrada más rápido que el material virgen $\left(455^{\circ} \mathrm{C}\right)$, posiblemente debido a que ya ha sufrido degradación durante el proceso de inyección de las tapas recicladas.

Tabla 9: Propiedades físicas del PPR

\begin{tabular}{l|c}
\hline \multicolumn{1}{c|}{ PROPIEDAD } & VALOR HALLADO \\
\hline Índice de fluidez & $2,7 \mathrm{~g} / 10 \mathrm{~min}$ \\
\hline Temperatura de degradación & $445^{\circ} \mathbf{C}$ \\
\hline Temperatura de transición vítrea $\left(\mathrm{T}_{\mathrm{g}}\right)$ & $-22,4^{\circ} \mathrm{C}$ \\
\hline Temperatura de fusión $\left(\mathrm{T}_{\mathrm{f}}\right)$ & $167,5^{\circ} \mathrm{C}$ \\
(rango de fusión) & $\left(95,4^{\circ} \mathbf{C}-201,6^{\circ} \mathbf{C}\right)$ \\
\hline Grado de cristalinidad $\left(\mathrm{X}_{\mathrm{c}}\right)$ & $45 \%$ \\
\hline
\end{tabular}

Los ensayos de calorimetría diferencial de barrido del PPR se realizaron con dos etapas de calentamiento y una de enfriamiento. El primer calentamiento permitió eliminar la historia térmica y las tensiones en la muestra y el segundo permitió determinar la temperatura de transición vítrea $\left(\mathrm{T}_{\mathrm{g}}\right)$, la temperatura de fusión $\left(\mathrm{T}_{\mathrm{f}}\right)$, la variación de entalpía de fusión $\left(\Delta \mathrm{h}_{\mathrm{f}}\right)$ y calcular, con ésta última, la cristalinidad $\left(\mathrm{X}_{\mathrm{c}}\right)$ empleando la ecuación (4).

$$
X_{C}=\frac{\Delta h_{f}}{\Delta h_{f}^{0}} \times 100 \%
$$

En esta expresión, el valor de $\Delta \mathrm{h}_{\mathrm{f}}$ obtenido es $93,6 \mathrm{~J} / \mathrm{g}$ y $\Delta \mathrm{h}_{\mathrm{f}}^{\circ}$ es el calor de fusión del PP completamente cristalino y para el cálculo se tomó el valor de $209 \mathrm{~J} / \mathrm{g}$ [16].

Las propiedades que se tomaron como referencia para este estudio son las propiedades del PP-virgen empleado en la fabricación de las tapas con las que se simuló el reciclado [17].

\subsection{Determinación de los parámetros de fabricación del CPM por medio de moldeo por compresión}

El objetivo es determinar los parámetros de fabricación del CPM, listados en la Tabla 10, que permitan obtener las mejores propiedades para su uso en la industria. 
Tabla 10: Parámetros de fabricación del CPM obtenido a partir de PPR y MCR

\begin{tabular}{l|c|c|c}
\hline \multicolumn{1}{c|}{ PARÁMETROS } & DESIGNACIÓN & UNIDAD & $\begin{array}{c}\text { RANGO DE } \\
\text { ENSAYO }\end{array}$ \\
\hline Tamaño de partícula de la madera capirona & $\mathrm{MRx}{ }^{*}$ & $\mathrm{~mm}$ & MR1 a MR3 \\
\hline Cantidad de capirona recuperada & $\mathrm{MCR}$ & $\%$ & 30 a 50 \\
\hline Temperatura de calentamiento en el horno & $\mathrm{T}_{\mathrm{w}}$ & ${ }^{\circ} \mathrm{C}$ & 170 a 190 \\
\hline Tiempo de permanencia de la muestra en el horno & $\mathrm{t}_{\mathrm{w}}$ & $\mathrm{min}$ & 10 a 100 \\
\hline
\end{tabular}

\subsubsection{Parte I: Definición de la temperatura de calentamiento y el tiempo de permanencia en el horno}

Según la metodología experimental descrita, los parámetros de partida considerados invariables están listados en la Tabla 11. En el apartado 2.2 se presentó la justificación de dichos valores.

Algunos parámetros, como la fuerza ejercida por la prensa y el tiempo de compresión durante el enfriamiento, fueron también constantes durante el moldeo de todas las muestras del estudio. A partir de experiencias anteriores, se ha establecido la fuerza de compresión en $34 \mathrm{kN}$ y el tiempo de enfriamiento forzado con aire en $10 \mathrm{~min}$ [18].

Tabla 11: Parámetros fijos en el proceso de fabricación del CPM, Parte I.

\begin{tabular}{l|c}
\hline \multicolumn{1}{c|}{ PARÁMETRO } & VALOR \\
\hline Tamaño de partícula de MCR & MR2 \\
\hline Porcentaje de partículas de MCR en volumen & $30 \%$ \\
\hline Porcentaje de PPR en volumen & $70 \%$ \\
\hline Fuerza de compresión & $34 \mathrm{kN}$ \\
\hline Tiempo de enfriamiento forzado con aire & $10 \mathrm{~min}$ \\
\hline
\end{tabular}

Las temperaturas de calentamiento se establecieron en el rango de $167,5^{\circ} \mathrm{C}$ a $201,6^{\circ} \mathrm{C}$ correspondiente al pico y al final de la fusión del PPR, definido con los resultados del ensayo DSC mostrados en la Tabla 9.

Para la Parte I se obtuvieron un total de 63 muestras debido a la inclusión de una temperatura adicional a las tres propuestas al inicio, con el propósito de tener un panorama más completo de las tendencias de la temperatura en función del tiempo.

Con la finalidad de seleccionar los parámetros óptimos del proceso de sinterizado, se ha realizado el ensayo de flexión a las 63 muestras del CPM obtenidas. En las Figuras 3 y 4 se visualizan las tendencias de la resistencia a la flexión y del módulo elástico en flexión respectivamente, para temperaturas de calentamiento entre $170^{\circ} \mathrm{C} \mathrm{y} 190^{\circ} \mathrm{C}$ a diferentes tiempos de permanencia en el horno $\left(\mathrm{t}_{\mathrm{w}}\right)$. Se puede apreciar que para cada temperatura existe un valor máximo de $\mathrm{R}_{\mathrm{f}} \mathrm{y} \mathrm{E}_{\mathrm{f}}$. Asimismo, los tiempos correspondientes a dichos máximos serán menores a mayores temperaturas de calentamiento. El menor tiempo observado para alcanzar los valores máximos, $\mathrm{R}_{\mathrm{f} \text { max }}=35,0 \mathrm{MPa} \mathrm{y} \mathrm{E}_{\mathrm{f} \text { max }}=2100 \mathrm{MPa}$, es $\mathrm{t}_{\mathrm{w}}=50$ min a Tw $=190^{\circ} \mathrm{C}$.

A partir de estos resultados, y en términos prácticos, si se desea reducir el tiempo de proceso se podría incrementar la temperatura de calentamiento $\left(\mathrm{T}_{\mathrm{w}}>190^{\circ} \mathrm{C}\right)$ hasta el límite establecido por la degradación de la madera $\left(\mathrm{T}_{\mathrm{w}}<240^{\circ} \mathrm{C}\right)$. 


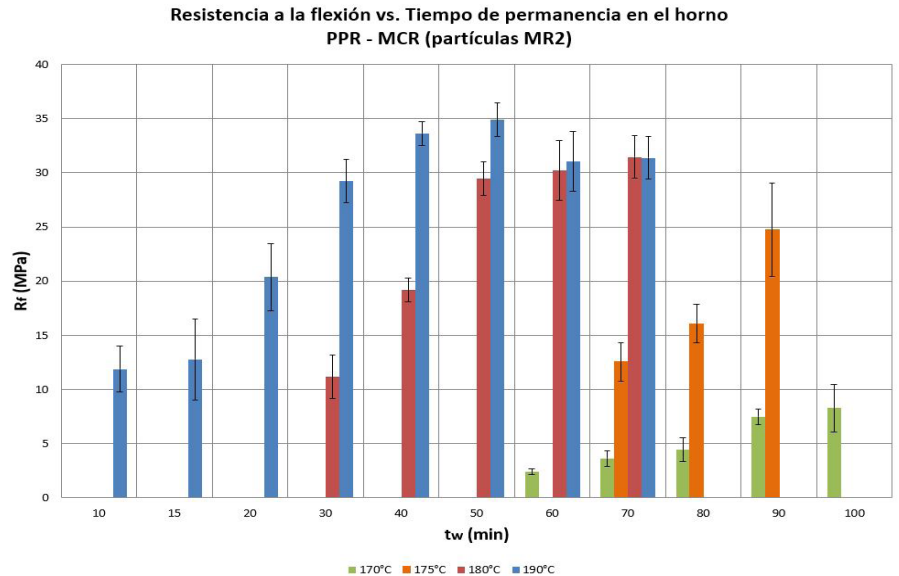

Figura 3: Resistencia a la flexión vs. tiempo de permanencia en el horno para diferentes temperaturas de calentamiento del CPM de PPR y MCR con tamaño de partícula MR2.

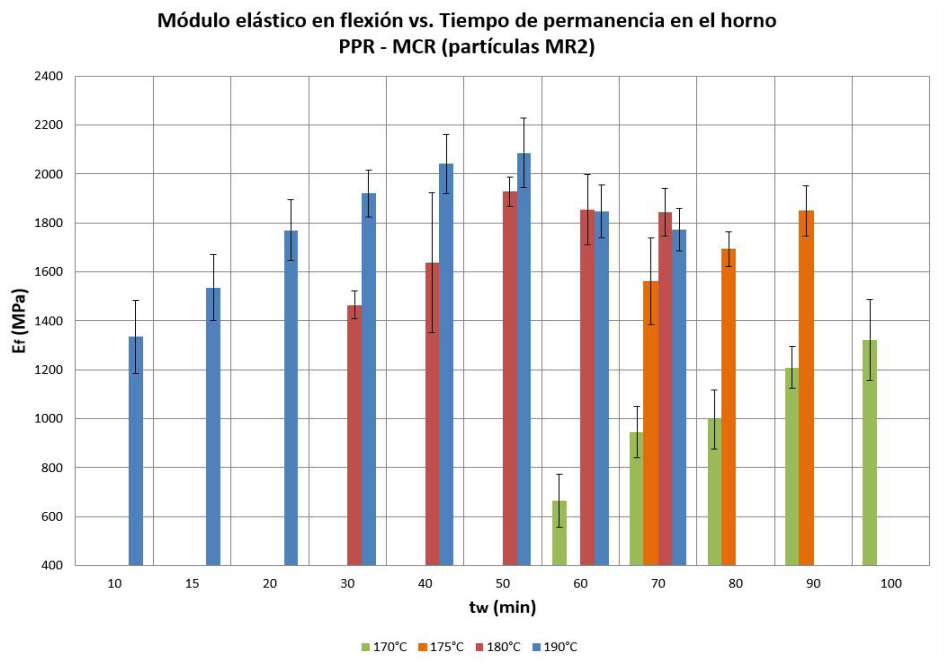

Figura 4: Módulo elástico en flexión vs tiempo de permanencia en el horno para diferentes temperaturas de calentamiento del CPM de PPR y MCR con tamaño de partícula MR2.

Desde otro punto de vista, es importante analizar el proceso de sinterizado y su influencia sobre las propiedades mecánicas. Inicialmente, mediante observación visual de las muestras fracturadas que fueron obtenidas a $\mathrm{Tw}=190^{\circ} \mathrm{C}$, se pudo apreciar que aquellas correspondientes a un $\mathrm{t}_{\mathrm{w}}=10$ min mostraban solidez superficial no obstante internamente las partículas de PPR y MCR aún no estaban adheridas; esto se observó también en muestras obtenidas a la misma temperatura para tiempos de 20, 30 y 40 min, aunque cada vez en menor grado. Con ello se concluye que el proceso de sinterizado del CPM $\mathrm{a} \mathrm{Tw}=190^{\circ} \mathrm{C}$ se completa recién a $\mathrm{t}_{\mathrm{w}} \geq 50 \mathrm{~min}$, en concordancia con la tendencia analizada de las propiedades mecánicas.

Posteriormente, utilizando micrografía SEM, se observaron muestras de CPM fabricados a $\mathrm{T}_{\mathrm{w}}=190^{\circ} \mathrm{C}$ y a diversos $\mathrm{t}_{\mathrm{w}}(30,40,50$ y $60 \mathrm{~min})$. Como resultado se encontró que en las muestras a $\mathrm{t}_{\mathrm{w}}<50$ min el polímero no embebió completamente a la madera, presentando cavidades y vacíos entre la matriz y el refuerzo. Esta situación explica las bajas propiedades resultantes a 30 y 40 min y que el proceso de sinterizado no ha concluido. En el caso del CPM con $\mathrm{t}_{\mathrm{w}} \geq 50$ min, se observó que el sinterizado ha terminado puesto que presenta una buena adhesión entre el plástico y la madera; aunque para 60 min se aprecian nuevos defectos cerca de la interfaz, posiblemente debido a la degradación de la madera, reduciendo significativamente la transferencia de carga y por tanto las propiedades mecánicas.

En conclusión, considerando el análisis de los valores máximos de las propiedades mecánicas y comprobando la adhesión entre las partículas de PPR y MCR mediante inspección visual y SEM como medios de verificación de un sinterizado completo, se seleccionaron como parámetros de trabajo de la Parte II de esta metodología: $\mathrm{T}_{\mathrm{w}}=190^{\circ} \mathrm{C} \mathrm{y} \mathrm{t}_{\mathrm{w}}=50$ min. Sin embargo, a partir de este resultado, se podría optimizar el tiempo de permanencia en el horno incrementando la temperatura de calentamiento, teniendo como límite la temperatura de inicio de la degradación del material compuesto. 


\subsubsection{Parte II: Definición del tamaño de partícula de madera y la proporción plástico-madera}

En la segunda parte experimental se realizaron ensayos en muestras de CPM fabricadas a $\mathrm{T}_{\mathrm{w}}=190^{\circ} \mathrm{C}$ y $\mathrm{t}_{\mathrm{w}}=50 \mathrm{~min}$, con la finalidad de establecer la proporción en volumen y el tamaño de partículas de madera capirona para obtener las mejores propiedades. Se fabricaron muestras variando el contenido en volumen de MCR entre 30\% y 50\% y los tamaños de partículas de madera en el rango comprendido entre $5,15 \mathrm{~mm}$ y $0,98 \mathrm{~mm}$. De ese modo se estudia la influencia de ambos parámetros en el comportamiento del material y el proceso de sinterizado.

\subsubsection{Análisis térmico y morfológico del CPM}

Mediante el análisis TGA se pudo observar que el CPM es más estable térmicamente que los materiales constituyentes por separado, al tener una temperatura de degradación mayor al del PPR y la MCR. Se observaron también dos etapas de degradación más marcadas a medida que se incrementa la cantidad de madera: la primera causada por la madera y la segunda por el plástico.

Del mismo modo se realizó el análisis DSC del material compuesto. En la Tabla 12 se presenta un resumen de los resultados, así como el porcentaje de cristalización calculado en cada caso. El porcentaje de cristalinidad disminuye a más de la mitad de la cristalinidad del PPR, lo cual también es coherente con la disminución de los valores de la entalpía de fusión.

Tabla 12: Resultados del DSC efectuado al CPM de PPR-MCR (con MCR de tamaño MR3)

\begin{tabular}{c|c|c|c}
\hline MUESTRA (\%PPR-\%MCR) & $\mathbf{T}_{\mathbf{F}}\left({ }^{\circ} \mathbf{C}\right)$ & $\mathbf{\Delta h}_{\mathbf{F}}(\mathbf{J} / \mathbf{g})$ & $\mathbf{X}_{\mathbf{c}}(\%)$ \\
\hline PPR & 167,5 & 93,60 & 44,78 \\
\hline $70 \%-30 \%$ & 161,2 & 33,98 & 21,89 \\
\hline $60 \%-40 \%$ & 161,9 & 35,77 & 26,34 \\
\hline $50 \%-50 \%$ & 164,1 & 26,50 & 22,94 \\
\hline
\end{tabular}

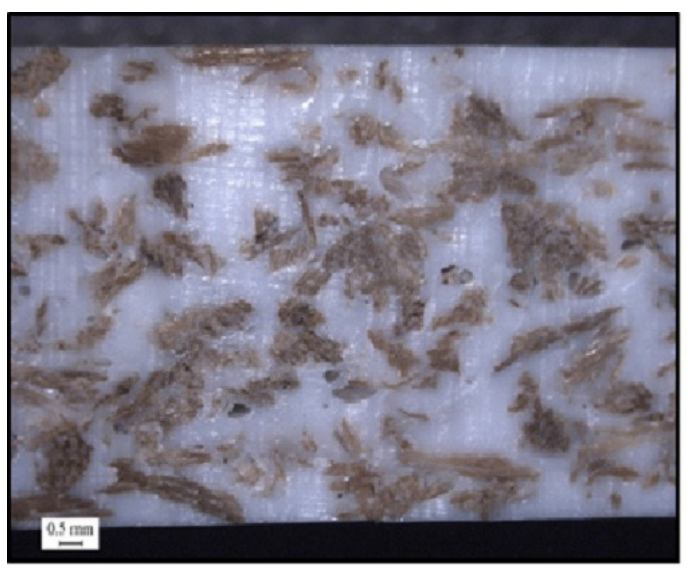

(a)

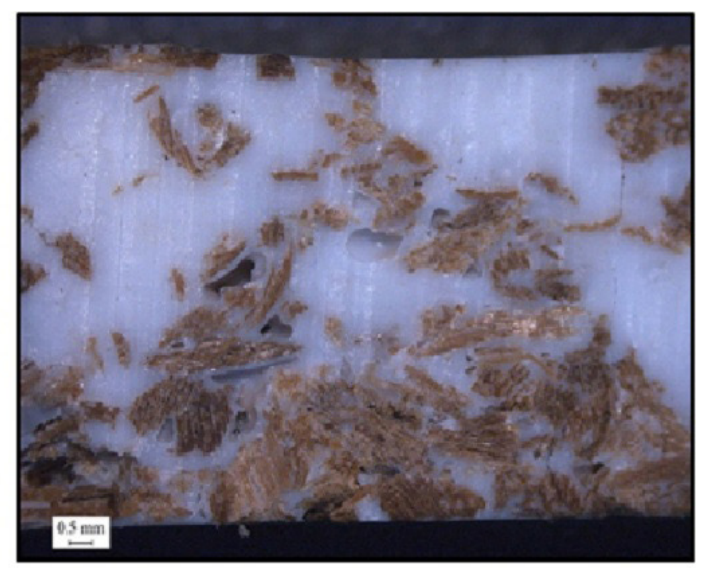

(b)

Figura 5: Sección transversal de muestras de CPM con partículas de capirona de tamaño MR3 (0,98 mm - 1,90 mm): a) $70 \%-30 \%$ de PPR-MCR (8X) y b) 50\%-50\% de PPR-MCR ( $8 \mathrm{X})$.

En el análisis FTIR realizado al material compuesto y a sus constituyentes se puede observar, al comparar los patrones y los picos referenciales de los materiales, que no existen coincidencias que indiquen una posible afinidad química entre PP y MC. Esto último es importante ya que el grado de adhesión entre PP y MC depende de ello.

En la Figura 5 se observan las secciones transversales de muestras con partículas de madera de tamaño MR3 y un contenido de madera del 30\% en la Figura 5(a) y de 50\% en la Figura 5(b). A partir de éstas se puede evidenciar que a mayor contenido de madera capirona se incrementa el número y tamaño de poros. Además, se puede apreciar que a mayor cantidad de madera la mezcla es más homogénea, aunque la matriz polimérica es más discontinua. Cabe mencionar que la falta de homogeneidad de la mezcla encontrada en las muestras observadas posiblemente se deba a que las partículas de termoplástico empleadas fueron más grandes (alrededor de los 5,0 $\mathrm{mm}$ ) que las de la madera; se constata que esta diferencia es mayor en las muestras de CPM fabricadas con partículas MR2 y MR3. 


\subsubsection{Ensayo de flexión}

En la Figura 6 se presentan los resultados del ensayo de flexión. Se muestra la resistencia a la flexión en la Figura 6(a) y el módulo elástico en la Figura 6(b) con diferentes tamaños de partícula de MCR y diferentes proporciones de PPR-MCR, incluido el PPR sin madera con fines comparativos.

Se observa que la resistencia a la flexión del CPM en todos los casos es menor a la del PPR (43,72 MPa) y mayor a la de la madera capirona en la dirección transversal (7,60 MPa, Tabla 8). Además, para cada tamaño de partícula, se puede apreciar que la resistencia a la flexión disminuye al aumentar la cantidad de madera. Este resultado es coherente en tanto que a menor cantidad de PPR menor será la resistencia.

Si se comparan las resistencias a una misma proporción PPR-MCR y diferentes tamaños de partículas, se observa que para la proporción 70\%-30\% se presenta, como se esperaba, un ligero incremento a menor tamaño de partícula de MCR. Sin embargo, dicha tendencia no se presenta en las proporciones $60 \%-40 \%$ ni $50 \%-50 \%$, en las que contrariamente disminuye el valor de la resistencia a menor tamaño de partícula de MCR. Esta disminución posiblemente se debe a la falta de homogeneidad en la mezcla debido a la diferencia de tamaños de partículas de MCR y PPR, mencionada en el análisis morfológico, que produce mayor discontinuidad en la matriz, además de la presencia de poros y vacíos según se observó en la Figura 5(b). Otra de las razones podría ser la falta de adhesión entre el PPR y la MCR, tal como se pudo prever gracias al análisis FTIR (ver 3.3.2.1), aun cuando se observa un buen embebido de las partículas.

Como se esperaba, para cada proporción de plástico-madera, $\mathrm{E}_{\mathrm{f}}$ aumenta ligeramente a medida que el tamaño de partícula disminuye. Por otro lado, para cada tamaño de partículas de madera, se observa un incremento del módulo elástico hasta un contenido de madera del $40 \%$ en volumen. Contrariamente a lo esperado, $\mathrm{E}_{\mathrm{f}}$ disminuye para un contenido de $50 \%$ de MCR; lo cual es posible que se deba a las mismas causas enunciadas sobre la disminución de $\mathrm{R}_{\mathrm{f}}$.

Finalmente, se observa que el CPM, en todos los casos, presenta un valor mayor de $\mathrm{E}_{\mathrm{f}}$ que el del PPR (1740 MPa) y mayor que $\mathrm{E}_{\mathrm{f}}$ en la dirección transversal de la madera capirona (1260 MPa, Tabla 8).

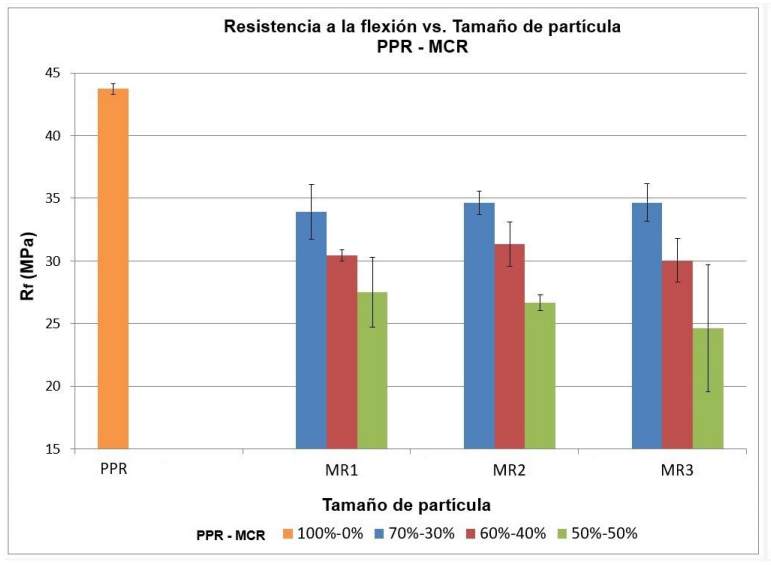

(a)

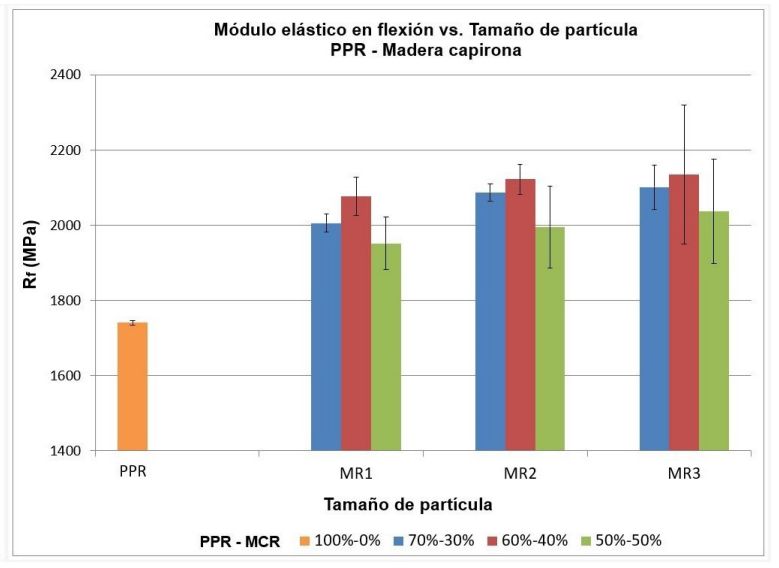

(b)

Figura 6: Ensayo de Flexión: a) Resistencia a la flexión vs tamaño de partícula de capirona y b) módulo de elasticidad en flexión vs tamaño de partícula de capirona, para diferentes proporciones de PPR-MCR.

\subsubsection{Ensayo de tracción}

En la Figura 7 se observan los resultados del ensayo de tracción del CPM: resistencia a la tracción en la Figura 7(a) y módulo elástico en la Figura 7(b) para diferentes tamaños de partícula de MCR y diferentes proporciones de PPR-MCR, incluido el PPR sin madera con fines comparativos.

Se observa que la resistencia a la tracción del CPM en todos los casos es menor a la del PPR (31,4 MPa). Asimismo, se pueden apreciar las mismas tendencias evidenciadas en el ensayo de flexión; siendo éstas coherentes, de manera particular para los materiales compuestos plástico-madera, ya que la presencia de poros, defectos o espacios vacíos en la interfaz plástico-madera influye negativamente y en mayor grado bajo cargas de tracción [19].

Sobre los resultados del módulo de elasticidad, se aprecia que el módulo del CPM siempre es mayor al módulo en tracción del PPR (1974 MPa). Para un mismo tamaño de partículas, como era de esperar, el valor de $\mathrm{E}_{\mathrm{t}}$ aumenta a medida que se incrementa la cantidad de MCR, debido a que la mayor rigidez de la madera promueve el incremento de la rigidez del CPM. 
A una misma proporción de PPR-MCR, se aprecia similarmente un ligero incremento en $\mathrm{E}_{\mathrm{t}}$ al disminuir el tamaño de partículas de MCR. En este caso, como en el ensayo de flexión, también era de esperar un significativo incremento de $\mathrm{E}_{\mathrm{t}} \mathrm{a}$ menor tamaño de partículas de madera; sin embargo, por las mismas razones que en el caso de la disminución de $\mathrm{R}_{\mathrm{f}}$, este incremento de $\mathrm{E}_{\mathrm{t}}$ es producto de un sinterizado ineficiente.

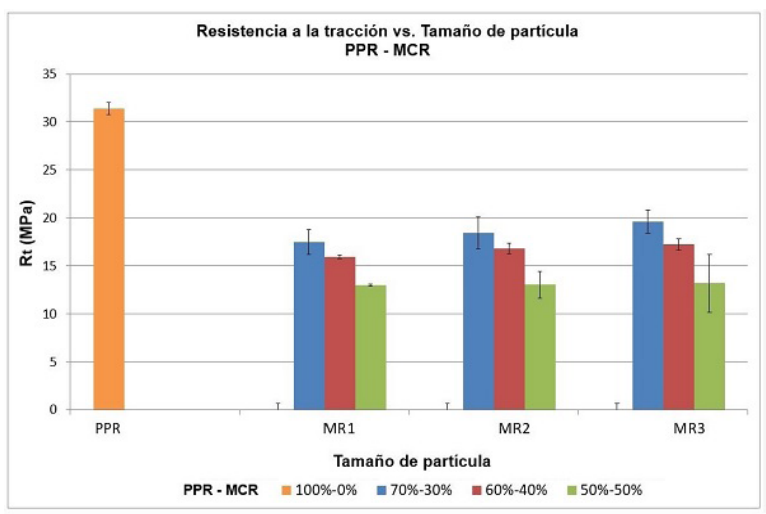

(a)

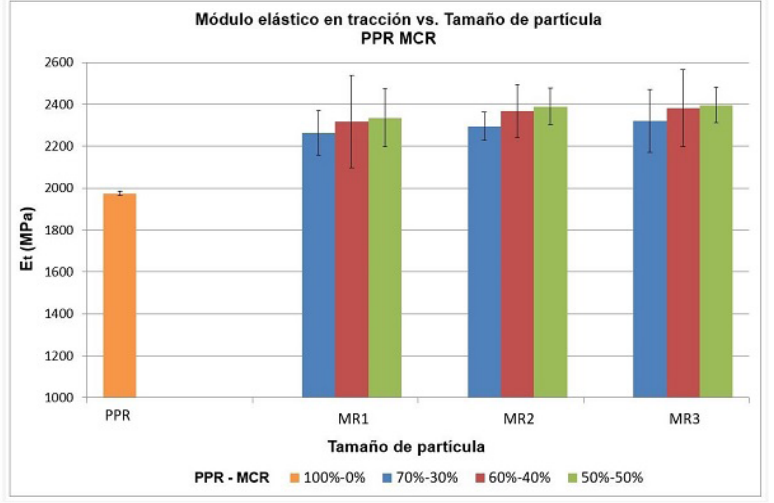

(b)

Figura 7: Ensayo de tracción: a) Resistencia a la tracción vs tamaño de partícula de capirona y b) módulo de elasticidad en tracción vs tamaño de partícula de capirona para diferentes proporciones de PPR-MCR.

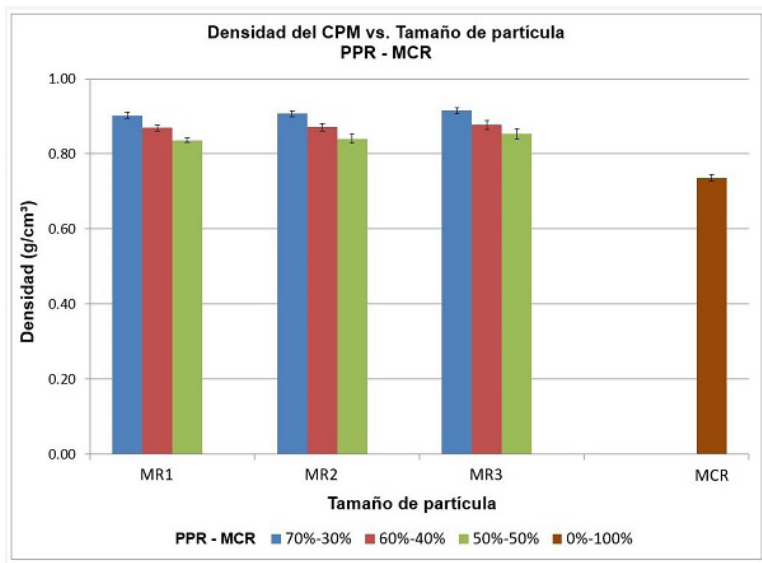

(a)

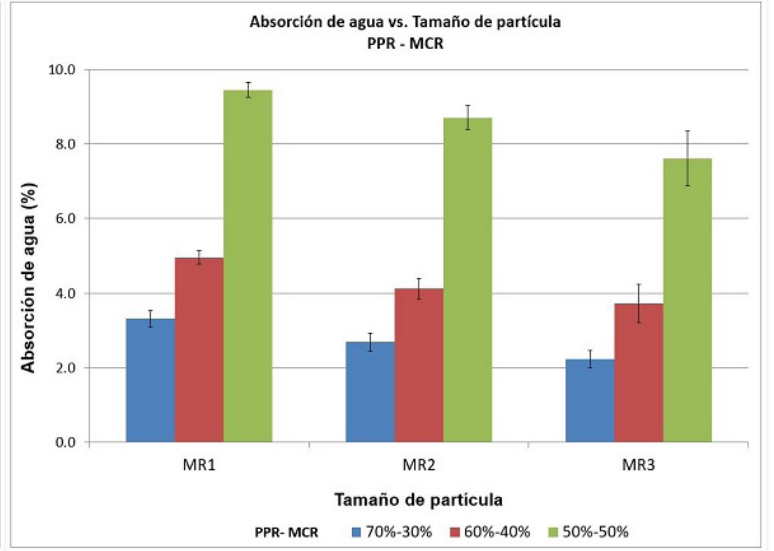

(b)

Figura 8: Ensayos de propiedades físicas del CPM: a) densidad y b) porcentaje de absorción de agua vs tamaño de partícula de capirona para diferentes proporciones de PPR-MCR.

\subsubsection{Ensayo de densidad}

Los valores de densidad obtenidos para el CPM se muestran en la Figura 8(a); se puede apreciar que, para cada tamaño de partículas de madera y a mayor contenido de MCR, el CPM presenta menor densidad por ser la madera capirona más liviana que el polipropileno. Se observa también que la densidad no varía apreciablemente con el tamaño de partículas de MCR para una misma proporción PPR-MCR y que, en todos los casos, el CPM presenta una mayor densidad que la de la madera capirona $\left(0,74 \mathrm{~g} / \mathrm{cm}^{3}\right)$ y menor que la del polipropileno virgen $\left(0,91 \mathrm{~g} / \mathrm{cm}^{3}\right)$, lo cual era previsible de acuerdo con la regla de las mezclas.

\subsubsection{Ensayo de absorción de agua}

En la Figura 8(b) se puede apreciar que el mayor porcentaje de absorción de agua de todas las muestras se presenta para el tamaño MR1 y una proporción 50\%PPR-50\%MCR, mientras que la mínima se consigue con partículas MR3 y una proporción 70\%PPR-30\%MCR. Este resultado se explica porque a más cantidad de madera capirona, que es un material higroscópico, la absorción del CPM será mayor. Asimismo, a mayor tamaño de partículas, se presentan más poros y vacíos en las interfaces, lugares donde se retiene agua. 
Se ha determinado que el porcentaje de absorción de agua obtenido para muestras de PPR fue de $0,063 \%$; resultado esperado en tanto que el PP es un material hidrofóbico y cercano al valor obtenido por otros investigadores para el mismo polímero [20]. Además, se puede deducir que los valores de absorción de agua del CPM en todos los casos están muy por encima del valor correspondiente al PPR y, a su vez, son menores que la absorción de agua de MCR (42,8\%) debido a su naturaleza hidrofílica.

Según ASHORI [21], una de las principales causas que favorecen la absorción de agua en los materiales compuestos plástico-madera son los vacíos interfaciales, los cuales se presentan en mayor número y tamaño en las muestras fabricadas con mayor tamaño de partícula y con mayores cantidades de MCR; esto último concuerda con los resultados obtenidos.

\subsection{Parámetros de fabricación del CPM para mejores propiedades}

En conclusión, y realizando un balance general de acuerdo con todos los resultados analizados, las mejores propiedades mecánicas y físicas las presenta el material compuesto fabricado con $70 \%$ de PPR y $30 \%$ de MCR con partículas de madera mayores a $0,98 \mathrm{~mm}$ y menores o iguales a $1,90 \mathrm{~mm}(\mathrm{MR} 3)$.

Finalmente, para el caso de CPM obtenido con partículas de PPR y MCR, podemos afirmar que las mejores propiedades se consiguen con los parámetros listados en la Tabla 13.

Tabla 13: Parámetros de fabricación del CPM de PPR y MCR por medio de moldeo por compresión para mejores propiedades. Parámetros con $\left(^{*}\right)$ fueron considerados fijos durante todo el estudio.

\begin{tabular}{l|c|c}
\hline \multicolumn{1}{c|}{ PARÁMETRO } & VALOR & UNIDADES \\
\hline Tamaño de partícula de la madera capirona (TP) & $0,98<\mathrm{TP} \leq 1,90$ & $\mathrm{~mm}$ \\
\hline Cantidad de capirona recuperada (MCR) & 30 & $\%$ \\
\hline Cantidad de polipropileno reciclado (PPR) & 70 & $\%$ \\
\hline Temperatura de calentamiento $\left(\mathrm{T}_{\mathrm{w}}\right)$ & 190 & ${ }^{\circ} \mathrm{C}$ \\
\hline Tiempo de permanencia de la muestra en el horno $\left(\mathrm{t}_{\mathrm{W}}\right)$ & 50 & $\mathrm{~min}$ \\
\hline Fuerza de compresión en el molde durante enfriamiento $^{*}$ & 34 & $\mathrm{kN}$ \\
\hline Tiempo de enfriamiento* $^{*}$ & 10 & $\mathrm{~min}$ \\
\hline
\end{tabular}

\section{CONCLUSIONES}

Se ha diseñado una metodología experimental general que permite determinar los parámetros de proceso para la obtención por sinterizado mediante moldeo por compresión de un material compuesto plástico madera (CPM), constituido de partículas de plástico reciclado (PR) y madera recuperada (MR), de tal manera que se alcancen las mejores propiedades para su utilización en la industria. Esta metodología consta de dos etapas: 1) Definición de la temperatura de calentamiento y el tiempo de permanencia en el horno y 2) Definición del tamaño de partícula de madera y la proporción plástico-madera.

Al aplicar dicha metodología en la fabricación de CPM a partir de partículas de polipropileno reciclado (PPR) y madera capirona recuperada (MCR), se han determinado los parámetros de proceso con los cuales se obtienen las mejores propiedades en dicho producto. Los parámetros encontrados fueron: Tamaño de partículas de la madera capirona recuperada entre 0,98 mm y 1,90 mm; proporción de PPR-MCR del 70\%-30\% en volumen; temperatura de calentamiento igual a $190^{\circ} \mathrm{C}$; tiempo de permanencia en el horno $50 \mathrm{~min}$; fuerza de compresión aplicada en el molde durante el enfriamiento de $34 \mathrm{kN}$ y tiempo de enfriamiento de $10 \mathrm{~min}$. A partir de estos resultados, se podría optimizar el tiempo de permanencia en el horno incrementando la temperatura de calentamiento, teniendo como límite la temperatura de inicio de la degradación del material compuesto.

Las propiedades obtenidas en el CPM moldeado bajo estos parámetros fueron: resistencia a la flexión $35 \mathrm{MPa}$; módulo de elasticidad en flexión $2100 \mathrm{MPa}$; resistencia a la tracción $20 \mathrm{MPa}$; módulo elástico a la tracción $2321 \mathrm{MPa}$; densidad $0,86 \mathrm{~g} / \mathrm{cm}^{3}$ y porcentaje de absorción de agua $2,2 \%$.

\section{AGRADECIMIENTOS}

Los autores agradecen al Laboratorio de Materiales (CITE) y al Laboratorio de Manufactura de la Sección Ingeniería Mecánica de la Pontificia Universidad Católica del Perú por el soporte técnico durante los ensayos de muestras y la fabricación de probetas y equipos respectivamente. Además, los autores agradecen a Innóvate-Perú por el financiamiento y apoyo a este trabajo de investigación (contrato N²01-FINCyT-IA-2013). 


\section{BIBLIOGRAFÍA}

[1] BIRON, M., "Thermoplastic Processing," In: Biron, M., Thermoplastics and Thermoplastic Composites., 2 ed., chapter 5, in Plastics Design Library, William Andrew Publishing, 2013.

[2] KORONIS, G., SILVA, A., FONTUL, M., “Green composites: A review of adequate materials for automotive applications," Compos. Part B Eng., v. 44, n. 1, pp. 120-127, 2013.

[3] ISAYEV, A.I., Injection and compression molding fundamentals, vol. 15. New York, Marcel Dekker INC., 1987.

[4] GERMAN, R., Sintering: from Empirical Observations to Scientific Principles. Elsevier, 2014.

[5] THÜMMLER, F., THOMMA, W.,"The sintering process”, Metall. Rev., v. 12, n. 1, pp. 69-108, 1967.

[6] SCRIBBEN, E., BAIRD, D., WAPPEROM, P., "The role of transient rheology in polymeric sintering”, Rheol. Acta, v. 45, n. 6, pp. 825-839, 2006.

[7] PRITCHARD, G.,"Two technologies merge: Wood-plastic composites”, Reinforced Plastics. pp. 26-29, 2004.

[8] BALUARTE-VASQUEZ, J.R., NEBEL, G., "Incremento diamétrico de Guarea macrophylla y Calycophyllum spruceanum en bosques inundables en la amazonía peruana”, Folia Amaz., v. 13, n. 1-2, p. 109, Jan. 2006.

[9] SIBILLE, A.M., “Guía de procesamiento industrial: Fabricación de muebles con maderas poco conocidas.," Lima, 2006.

[10] SIMPSON, W.T., "Equilibrium Moisture Content of Wood in the United States and Worldwide," Forest Products Journal, no. FPL-RN-0268. Madison, Wisconsin, p. 11, 1998.

[11] GAGO, J., LEÓN, K., RAMOS, V., et al. "Desarrollo de un material compuesto a base de residuos de madera Capirona ( Calycophyllum spruceanum ) y polietileno," in Informe Científico Tecnológico 2010, Ed. Lima: Instituto Peruano de Energía Nuclear, pp. 43-50, 2011.

[12] MEHROTRA, R., SINGH, P., KANDPAL, H.,"Near infrared spectroscopic investigation of the thermal degradation of wood," Thermochim. Acta, v. 507-508, pp. 60-65, 2010.

[13] SIERRA, S.A., Estudio de materiales compuestos de matriz polimérica y refuerzos de partículas de madera obtenidos a partir de polipropileno reciclado y capirona recuperada, Tesis de Magister, Pontificia Universidad Católica del Perú, Lima, Perú, 2012.

[14] KACZMAREK, H., OLŁDAK, D., MALANOWSKI, P., et al. "Effect of short wavelength UV-irradiation on ageing of polypropylene/ cellulose compositions," Polym. Degrad. Stab., v. 88, n. 2, pp. 189-198, 2005.

[15] LYONDELLBASELL INDUSTRIES HOLDINGS, "Polypropylene, Homopolymer (Lyondellbasell)," 2018. [Online]. Disponible en:https://www.lyondellbasell.com/en/products-technology/polymers/resin type/polypropylene-homopolymer/. [Consultado: 09-Jul-2018].

[16] BUTYLINA, S., HYVÄRINEN, M., KÄRKI, D.T., “A study of surface changes of wood-polypropylene composites as the result of exterior weathering," Polym. Degrad. Stab., v. 97, n. 3, pp. 337-345, 2012.

[17] POLIPROPILENOS DEL CARIBE S.A., PROPILCO 02H84ND, web, 2009. [Online]. Disponible en: http://ptrasandina.com.ve/wp-content/uploads/productos/propilco/homopolimeros_ht/BT_ES_02H84ND.pdf. [Consultado: 09-Jul-2018].

[18] BRAÑEZ, L.E., ARRIBASPLATA, A.S., TUPIA, W.M., et al. "Determinación de los parámetros de moldeo por compresión de materiales compuestos fabricados con madera capirona (Calycophyllum Spruceanum) recuperada y polipropileno reciclado,” In: XIII Congreso Iberoamericano de Ing.Mecánica, Lisboa, Portugal, Oct. 2017.

[19] WARD-PERRON, N., RODRIGUE, D., "Natural Fiber Reinforced Thermoplastics (Nfrtp) Processed By Rotomolding," 70th Annu. Tech. Conf. Soc. Plast. Eng. 2012 (ANTEC 2012), pp. 2263-2267, 2012.

[20] MAT-SHAYUT, M.S., ABDULLAH, M.Z., MEGAT-YUSO, P. S., "Water Absorption Properties and Morphology of Polypropylene/ Polycarbonate/Polypropylene-graft-Maleic Anhydride Blends,” Asian J. Sci. Res., v. 6, n. 2, pp. 167-176, 2013.

[21] ASHORI, A., "Wood-plastic composites as promising green-composites for automotive industries!," Bioresource Technology, v. 99, n. 11. pp. 4661-4667, 2008.

\section{ORCID}

Walter Mariano Tupia Julio Arnaldo Acosta Adan Smith Arribasplata

Luz Elena Brañez Haro http://orcid.org/0000-0003-3432-7178 https://orcid.org/0000-0002-1518-4282

https://orcid.org/0000-0002-2978-1926 https://orcid.org/0000-0003-1684-8519 